\title{
Selection of a Suitable Agile Methodology - Case Study
}

\author{
František HAŠEK and Hana MOHELSKÁ* \\ University of Hradec Králové, Hradec Králové, Czech Republic; frantisek.hasek@uhk.cz; \\ hana.mohelska@uhk.cz \\ * Corresponding author: hana.mohelska@uhk.cz
}

\begin{abstract}
Lean manufacturing and lean thinking, in general, have accompanied especially manufacturing companies for many decades. This is not different when developing software. Agile development methodologies are currently among the most used methods in software development and their supply is relatively wide. At the same time, the selection of a suitable method is fundamental, not only for timely delivery but mainly for the optimal functionality of the entire final solution. The agile methodology is mainly focused on value for the customer. This paper is focused on a case study of selecting a suitable agile methodology for a selected medium-sized company. The selection criteria were based on a discussion with the key employees, and then the weights for the particular criteria were determined using a questionnaire survey. The attributes of the selected methodologies were evaluated by experts from the selected company. Saaty's method was chosen for multicriteria decision-making. Subsequently, a decision support system (Criterium Decision Plus) was used to select a suitable methodology from the selection of the most used agile methods.
\end{abstract}

Keywords: agile software development; scrum; extreme programming; kanban

JEL Classification: M10; M11; M15

\section{Introduction}

In the 1960s, the branch of system engineering experienced a rise for which, as it turned out, it was not prepared. The rapid development caused an increase in demand and that caused problems with extensions, increases in price, or project crashes. Software development has evolved in many phases, one of them is called "code and fix" or waterfall methods like "heavyweight", agile methods are considered like leading ones in the "lightweight" category. Each of these methods has been more or less successful, synergy between these approaches brings still better and better methods. (Oyong \& Ekong, 2019).

In today's world of globalization and the increasingly advanced digitalization of the world, the constant expansion of lean manufacturing and sustainability is essential, these aspects could be achieved by agile methodologies that need to be implemented across the organization, from management to development teams. (Dänner \& Doubek, 2020).

The term agile can be described as dynamic, limber or adaptable. Everyone in the team does what is just needed. Traditional software development techniques do not allow a later change of the assignment, while agile methodologies allow not only to modify the assignment during development but also to check and correct errors, thus becoming almost 
flawless (Neelu \& Kavitha, 2020). These methods are based on a frequent delivery of software, customer satisfaction, intensive cooperation with customers and requirements that can be changed anytime. However, there are several challenges, for example, scalability, smooth control of development or the ability for remote collaboration (Younas et al., 2019). The main features of agile methodologies are timely delivery of software, interaction with the customer, re-learning from previous iterations, operational changes at any stage of project development and so on. (Younas et al., 2019). To achieve the mentioned features, an agile manifesto was designed with four basic values (Manifesto for Agile Software Development, n.d.):

- individuals and interactions over processes and tools,

- working software over comprehensive documentation,

- customer collaboration over contract negotiation,

- responding to change over following a plan.

Users of agile software development methods appreciate the ability to manage changing priorities, project visibility, delivery speed or team morale. Scrum, Kanban, and Extreme programming belong to the world's most used agile methodologies in 2019. (State of Agile, n.d.).

\subsection{Extreme Programming}

The Extreme Programming methodology was introduced in 1999, also known as XP, and is one of the most efficient, light, flexible, and fun methods of software development. It is suitable for small and medium-sized teams that have unclear or variable assignments. This method is called extreme because it brings commonly used procedures and principles to extreme dimensions, where the team is constantly focused on one functional element. The key to the success of the XP method are four fundamental values: communication, simplicity, feedback and courage. Communication is essential in project management because many problems are caused by poor communication. With the help of simplicity, it is possible to implement functional changes in the shortest possible time without delay, which are modified and further improved thanks to the feedback. The last key method is courage, thanks to which fundamental steps and changes can be realized. The subliminal value is the respect for team members, without which XP could not succeed. (Beck \& Makovec, 2002).

\subsection{Kanban}

The roots of the Kanban method go back to Japan, where the method was used to regulate visitors in temples, where each visitor took one ticket at the entrance and returned it at the exit. The number of tickets was limited and thus the capacity of the temple. In project management, Kanban is used for efficient visualization using a Kanban board, which reduces work in progress and minimizes throughput, thus achieving faster delivery and better quality of software delivery. An example is a board divided into processes (e.g. To Do - In Progress - Done), in which taskbars are located. The maximum number of tickets on the board should be limited(Zayat \& Senvar, 2020). 


\subsection{Scrum}

The Scrum methodology is based on lean thinking and empiricism and it is used as a process framework for managing the development of complex products. This process consists of constant interaction within a team in which its members work together from the beginning to the end of development. For Scrum to work properly, it has to consist of Scrum teams and associated roles, activities, artifact, and rules. Scrum is defined as simple, easy to understand, but extremely difficult to master (Schwaber \& Sutherland, 2017).

Scrum teams are self-organizing teams that choose how the work will be done and that consist of the product owners, the development team, and the Scrum master. People in scrum are not divided into sub-teams or hierarchy and the maximum number of members of the Scrum team are around 10 because communication in smaller groups is much easier. Developers are people who develop any part of a product whose skills are usually broad. The product owner is responsible for maximization of value during working on the project, communication and management of backlog. Scrum Master's work is based on disseminating the know-how about theory and practice of the Scrum in the Scrum team and whole organization. (Schwabre \& Sutherland, 2020).

\section{Methodology}

To determine the weights of the selected criteria, a questionnaire survey was conducted, which took place within the company. The company that the case study focuses on belongs to the medium-sized companies, employs 90 people, and is focused on software development. Before the main survey, a pilot study was carried out in cooperation with the product director to verify the feasibility of the questionnaire and then the anonymous questionnaire was distributed to 10 employees interested in agile software development. The questionnaire contained 21 questions of pairwise evaluation of selected criteria.

As the method for multicriteria decision making, Saaty's method was chosen, which compares pairs of criteria with each other. Respondents were therefore asked to take this step and always evaluate the two criteria. The weights of the individual criteria were obtained using Saaty's matrix, and then the arithmetic mean for each criterion was determined from the different weights. The final weights are entered in Table 1.

Table 1. Final weights of the selected criteria

\begin{tabular}{|c|c|}
\hline Criterion & Weights \\
\hline $\begin{array}{c}\text { The degree of complexity } \\
\text { of the methodology }\end{array}$ & 0.031 \\
\hline $\begin{array}{c}\text { The degree of } \\
\text { documentation }\end{array}$ & 0.054 \\
\hline The degree of error & 0.227 \\
\hline $\begin{array}{c}\text { Specification of the } \\
\text { customer requirements }\end{array}$ & 0.315 \\
\hline Delivery time & 0.179 \\
\hline Defining responsibility & 0.068 \\
\hline $\begin{array}{c}\text { Communication across the } \\
\text { team }\end{array}$ & 0.126 \\
\hline$\sum$ & 1.000 \\
\hline
\end{tabular}


Respondents consider the specification of the customer requirements to be the most important criterion. They chose it because they perceive the correct understanding of the customer as the most important factor, with the incorrect specification of requirements, the work of the entire team will be in vain. The second most important criterion was the degree of error. As with the incorrect specification, the high error rate leads to inefficiency of the performed work. The effort to eliminate errors within the entire team is reflected in the perceived importance of this criterion. The third criterion according to the importance is the delivery time. The company should release a new version of the system without much time delay. Communication across the team finished in the fourth place and overtook the criteria for defining responsibility, the degree of documentation and the degree of complexity of the methodology. Communication is a key part of agile development. On the contrary, respondents chose as the least important criterion the degree of complexity of the methodology.

\section{Results}

Presently, several methods are used for agile development, the three most used will be assigned to individual criteria according to the suitability of the methodology. An expert opinion together with research were used for this selection. The selection included methodologies: Scrum, Kanban, and Extreme Programming (XP), these three mentioned methodologies were listed as the most used in 2019 (figure 1), with the exception of hybrid methodologies.

\subsection{Evaluation of Methods According to Criteria}

For the final selection of the methodology, the Criterium Decision Plus software was selected, in which the weights of the individual criteria were determined and below the methodologies will be evaluated based on the selected criteria. The opinion of an expert from the given company was used to evaluate the methodologies.

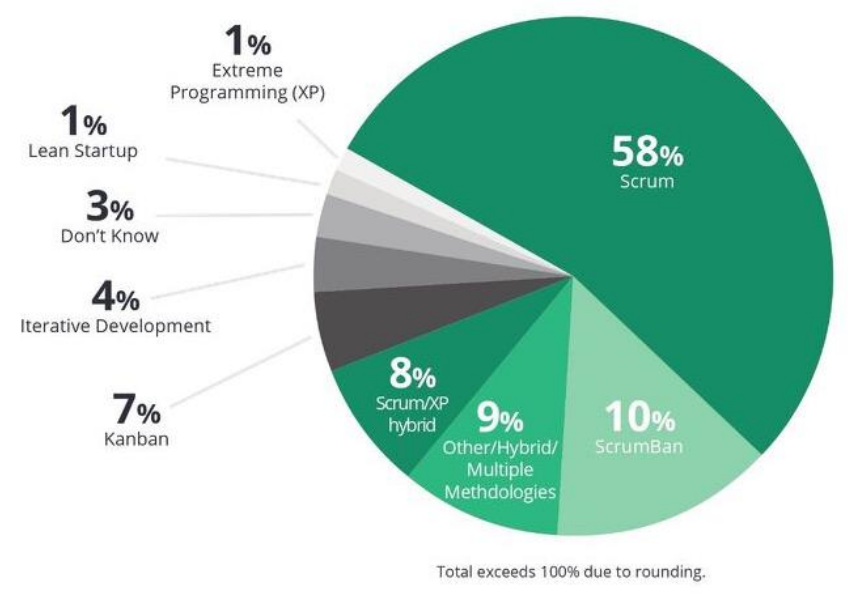

Figure 1. The agile methodology used in 2019 (State of Agile, n.d.) 


\section{The degree of complexity of the methodology}

Although the purpose of agile methodologies is simplicity, the Kanban methodology, which has its roots in lean manufacturing, was described as the simplest. The Kanban methodology in its basis does not dictate or prohibit anything, it only has to follow three principles: visualize the work, minimize the time of passage and limit the work in progress. The Scrum and XP methodologies have been identified as similarly complex, as they already have to follow certain procedures but they cannot be considered complicated.

\section{The degree of documentation}

Even though one basic value of the Agile Manifesto says that it prefers working software to exhaustive documentation, this does not mean that the documentation can be omitted altogether and no attention paid to it. The degree of documentation depends on the claims of the contracting authority. From this point of view, Extreme Programming turned out to be the most suitable method, as one of the twelve practices are the standards for the writing source text, which developers must adhere to. The source code represents part of the documentation in the XP methodology. Thanks to the established rules for writing code, it is not a problem to know the individual lines, even if they were written by someone else.

\section{The degree of error}

Some factors can affect the degree of error, whether it is the motivation of the employee or his abilities. The advantage of Extreme Programming is that it follows the practice of a forty-hour week, which contributes significantly to reduce errors. Thus, workers do not make mistakes that would stem from their fatigue.

\section{Specification of the customer requirements}

In traditional development, it has been common for customer requirements not to be well defined or for misunderstanding by the application manufacturer. This was mainly due to the insufficient communication between the two parties. Scrum defines the important role of the Product Owner, who is a part of the team and is responsible for individual system requirements. In the XP methodology, the customer is also assigned to the developer's workplace but does not have precisely defined tasks, he is only available to programmers. Therefore, the Scrum method was chosen as the best for the specification of the customer requirements.

\section{Delivery time}

With delivery time, the priority is not to delay the release of a new version. Scrum seems to be the most appropriate methodology, which divides development into multiple iterations (in the case of Scrum sprints), with working software at the end of each sprint to avoid delivery delays. The Kanban methodology has been placed right behind Scrum with the principle of minimizing passage time, so the delivery should be realized as soon as possible. Defining responsibility

All selected methodologies share responsibility within the whole team. When someone has a problem, it is not the individual's, but everyone's problem. However, the most suitable methodology is Scrum because the role of the product owner is responsible for all system requirements (Product Backlog). 


\section{Communication across the team}

Communication is one of the fundamental pillars of all agile methodologies. The methodologies were evaluated concerning the added value which communication offers. The Scrum methodology was rated the best, it includes a lot of meetings in the whole process, starting with planning and ending with a retrospective. However, daily meetings where members exchange information are most beneficial. Although the XP methodology does not set any meetings, it uses the practice of pair programming, in which programmers are in constant communication contact.

Table 2. Features of the methodologies for selected criteria

\begin{tabular}{|l|l|l|l|l|}
\hline Lowest Level & Scrum & Kanban & XP & Model \\
\hline The degree of complexity of the methodology & 0.170 & 0.660 & 0.170 & 0.031 \\
\hline The degree of documentation & 0.250 & 0.250 & 0.500 & 0.054 \\
\hline The degree of error & 0.200 & 0.200 & 0.600 & 0.227 \\
\hline Specification of the customer requirements & 0.530 & 0.170 & 0.300 & 0.315 \\
\hline Delivery time & 0.560 & 0.320 & 0.120 & 0.179 \\
\hline Defining responsibility & 0.500 & 0.250 & 0.250 & 0.068 \\
\hline Communication across the team & 0.540 & 0.160 & 0.300 & 0.126 \\
\hline
\end{tabular}

Based on the multi-criteria analysis and using the management tool for decision support Criterium Decision Plus, the resulting graph (figure 2) was compiled, which selects the most suitable agile methodology for implementation in the selected company.

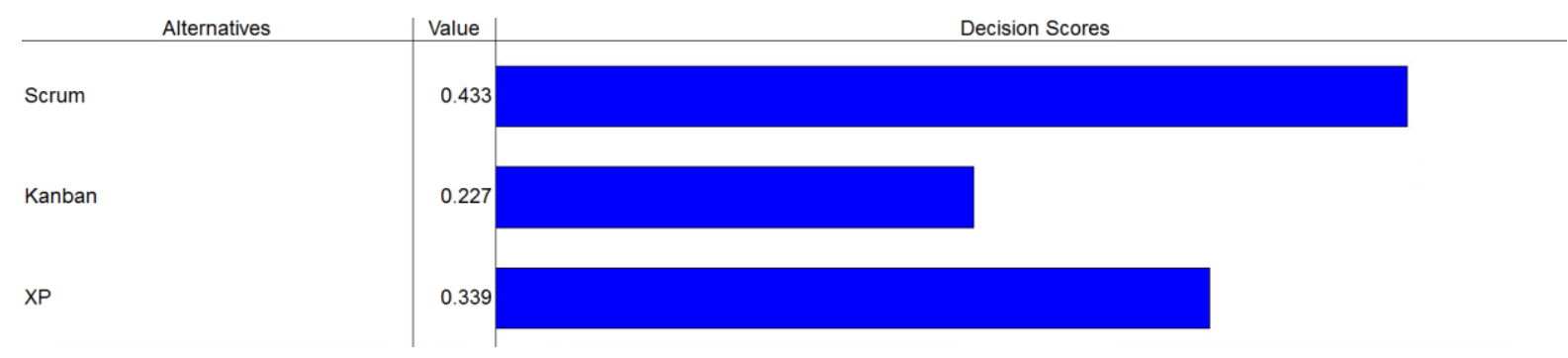

Figure 2. Graph of the selection of the optimal methodology

It is noticeable from the output that the Scrum method is the best choice. This happened mainly due to the more detailed elaboration of the methodology, its dominance is confirmed by the fact that it is the most used agile methodology in the world. The second position was taken by the XP (Extreme Programming) methodology. Kanban turned out to be the least suitable of the examined methodologies. It seems to be very abstract and is more suitable as a complementary methodology. In the next discussion, it would be appropriate to combine the two methodologies with the fact that the basic framework would be the Scrum methodology and would be enriched with some principles from another methodology.

\section{Conclusions}

For the objective assignment of the weights, a questionnaire survey was carried out, in which five employees (experts) performed a pairwise evaluation of selected criteria. From the results, the weights of individual criteria were determined with the help of Saaty's matrix, and these criteria were evaluated by selected agile methodologies. As a result, decision 
support system Criterium Decision Plus selected the most suitable agile methodology for implementation in the analyzed company, the Scrum method, which is the most used agile methodology in the world (the State of Agile, n.d.). Contribute to this result, Scrum is very flexible. For many companies, implementing an agile approach means increasing competitiveness.

Acknowledgments: The paper was written with the support of the specific project 2021 grant "Determinants of cognitive processes impacting the work performance" granted by the Faculty of Informatics and Management, the University of Hradec Králové, Czech Republic.

\section{References}

Beck, K., \& Makovec, P. (2002). Extrémní programování. Grada Publishing.

Dänner, K., \& Doubek, S. (2020). Factors Influencing the Success of "Agile Working" in Manufacturing Companies.

Manifesto for Agile Software Development. (n.d.). http://agilemanifesto.org/

Neelu, L., \& Kavitha, D. (2020). Software Development Technique for the Betterment of End User Satisfaction using Agile Methodology. TEM Journal, 9(3), 992-1002. https://doi.org/10.18421/TEM93-22

Oyong, S. B., \& Ekong, V. E. (2019). An explorative survey of formal and agile software development methods. Global Journal of Pure and Applied Sciences, 25(1), 71. https://doi.org/10.4314/gjpas.v25i1.10

Schwaber, K., \& Sutherland, J. (2017). The Scrum Guide. https://www.scrumguides.org/docs/scrumguide/v2017/2017-Scrum-Guide-US.pdf

Schwabre, K., \& Sutherland, J. (2020). The Scrum Guide. https://www.scrumguides.org/docs/scrumguide/v2020/2020-Scrum-Guide-US.pdf\#zoom=100

State of Agile. (n.d.). 15th Annual State of Agile Survey. https://stateofagile.com/\#ufh-i-615706098-14th-annualstate-of-agile-report/7027494

Younas, M., Jawawi, D. N. A., Ghani, I., Shah, M. A., Khurshid, M. M., \& Madni, S. H. H. (2019). Framework for Agile Development Using Cloud Computing: A Survey. Arabian Journal for Science and Engineering, 44(11), 8989-9005. https://doi.org/10.1007/s13369-019-03923-6

Zayat, W., \& Senvar, O. (2020). Framework Study for Agile Software Development Via Scrum and Kanban. International Journal of Innovation and Technology Management, 17(04), 2030002.

https://doi.org/10.1142/S0219877020300025 